LBNL-46878

\title{
REACTIVE SPREADING: ADSORPTION, RIDGING AND COMPOUND FORMATION
}

E. Saiz, R. M. Cannon and A. P. Tomsia

Materials Sciences Division
Lawrence Berkeley National Laboratory
University of California,
Berkeley, CA 94720 USA

May, 2000

Acta Materialia

Accepted

Work supported by the Director, Office of Energy Research, Office of Basic Energy Sciences, Materials Sciences Division of the U. S. Department of Energy under Contract No. DE-AC03$76 \mathrm{SF} 00098$. 


\title{
REACTIVE SPREADING: ADSORPTION, RIDGING AND COMPOUND FORMATION
}

\author{
E. Saiz, R. M. Cannon and A. P. Tomsia \\ Materials Science Division, Lawrence Berkeley National Laboratory, Berkeley, CA 94720, USA
}

\begin{abstract}
Reactive spreading, in which a chemically active element is added to promote wetting of noble metals on nonmetallic materials, is evaluated. Theories for the energetics and kinetics of the necessary steps involved in spreading are outlined and compared to the steps in compound formation that typically accompany reactive wetting. These include: fluid flow, active metal adsorption, including nonequilibrium effects, and triple line ridging. All of these can be faster than compound nucleation under certain conditions. Analysis and assessment of recently reported experiments on metal/ceramic systems lead to a focus on those conditions under which spreading proceeds ahead of the actual formation of a new phase at the interface. This scenario may be more typical than believed, and perhaps the most effective situation leading to enhanced spreading. A rationale for the pervasive variability and hysteresis observed during high temperature wetting also emerges.
\end{abstract}

\section{INTRODUCTION}

Most pure, noble metals (e.g., Pt, $\mathrm{Au}, \mathrm{Ag}, \mathrm{Cu}, \mathrm{Ni}$ ) exhibit obtuse contact angles on higher melting-point ceramics (such as $\mathrm{SiC}, \mathrm{Si}_{3} \mathrm{~N}_{4}$ or $\mathrm{Al}_{2} \mathrm{O}_{3}$ ) or graphite [1-6], and this hampers their use in applications like brazing, bonding, liquid-phase sintering, or infiltration. The angles range typically between $100^{\circ}$ and $150^{\circ}$ and increase roughly with the free energy of formation or band gap of the ceramic. This problem can be alleviated by alloying a small amount of a reactive metal (such as $\mathrm{Ti}, \mathrm{V}, \mathrm{Cr}, \mathrm{Zr}, \mathrm{Nb}, \mathrm{Hf}$, or even $\mathrm{Al}$ ) into the liquid. These additions can enhance wetting angles [2-4, 7-13], often strongly (Fig. 1). Because of their technological importance, a large body of empirical knowledge about the effects of reactive elements on spreading has been accumulated, but the results are complex, ambiguous and even inconsistent. Contact angles usually evolve unaccountably slowly over $10^{2}-10^{4}$ seconds $[2,10,11,21]$ (Fig. 2), ${ }^{1}$ and reaction products such as nitrides, silicides or oxides have frequently been found at or near the interface after solidification [7-11, 22]. However, recent experiments in which liquid drops are transferred directly to a hot substrate give spreading rates $[23,24]$ nearer those of low viscosity aqueous or organic fluids $[25,26]$ and far higher than that when the liquid-forming material is heated in place.

Controversy persists regarding the physicochemical mechanisms that control the wetting and spreading of such reactive alloys. Specifically, a predictive capability for the influence of bulk reactions on wetting behavior has not emerged. Some variational treatments of the problem led to the inclusion of a reaction energy term for an undefined thickness of reactive phase in the Young equation [28-30]; these treatments anticipated that wetting is superior when a compound forms simultaneously. However, none can predict the optimal additive or extent of reaction, much less the wide variability in wetting angles and spreading times nor can they predict cases of reactivity with poor wetting. Alternative considerations have focused on the effects of

\footnotetext{
${ }^{1}$ This figure contains previously unpublished data. Contacts angles were measured as described in Refs. [10, 11] and converted into velocities assuming evolution occurs as a series of constant curvature shapes.
} 
equilibrium adsorption on the solid/liquid interfacial energy as a determinant $[3,4,14,27]$. However, verification has been difficult owing to subsequent reactions and changes upon cooling. Little theory exists concerning the efficacious surface activity of elements. The coupling of fluid flow and degree of reactivity remains to be established in terms of kinetic mechanisms and identification of appropriate local driving forces for fluid motion.

\section{THEORETICAL CONCEPTS}

As a step toward developing such understanding, the present paper divides reactive spreading into a series of stages, depending on the time or velocity. These stages include fluid flow, adsorption of active element at the several interfaces, triple point ridging, and finally, formation of a new compound at the solid-liquid interface (Fig. 3). During wetting, a system will pass successively through various stages, but depending on the properties of a system, the time scale in which any observation is actually accomplished may correspond to only one of them. The idea is to determine which step controls spreading kinetics and what causes the decrease of contact angle. Moreover, the extent of reaction that occurs during fluid flow for a given system will depend strongly on the energetics and kinetics of compound formation. Thus, pertinent stages are briefly described. The goal is to analyze each in terms of the triple-junction structure and chemistry, the relevant surface energies that drive wetting, and the mechanisms that control spreading kinetics.

\subsection{Fluid flow}

Numerous theories have analyzed spreading in terms of one fluid displacing another over a solid surface that is effectively presumed to be rigid and insoluble. We denote this situation as regime I, with successive spreading regimes corresponding to increasing degrees of substrate deformation, and focus on a liquid/gas combination. The capillary forces drive a homogeneous liquid towards a shape of constant curvature and the contact angle towards the $\theta_{I D}$ value, that given by the Young-Dupré equation [31]:

$$
\gamma_{l v} \cos \theta_{I D}=\gamma_{s v}-\gamma_{s l}
$$

where $\gamma_{l v}$ and $\gamma_{s v}$ are the liquid and solid surface energies, and $\gamma_{s l}$ is the solid/liquid interfacial energy. Other driving forces from hydrostatic pressure gradients can be ignored here. Hysteresis (the fact that in many cases advancing and receding liquid fronts seem to arrive at different stationary contact angles) is usually explained in terms of morphological or chemical inhomogeneities in the substrate, or by the fact that for contact angles close to equilibrium, spreading occurs too slowly to be accurately recorded in practical experiments [26, 32-34].

Dynamic wetting has been analyzed both from the perspectives of continuum mechanics (hydrodynamics) [26, 33, 34] and microscopic or molecular mechanisms [32, 35, 36]. Hoffman [37] first postulated that for systems with perfect wetting $\left(\theta_{I D}=0\right)$ the macroscopic dynamic contact angle $\left(\theta_{D}\right)$ depends on the spreading velocity $v$ through the capillary number, where $C a \equiv$ $\eta v / \gamma_{l v}$ and $\eta$ is the liquid viscosity. This idea has been subsequently extended to systems with partial wetting. A popular expression for the relationship between contact angle and spreading velocity is the generalized Hoffman-Voinov-Tanner law, valid for small capillary numbers [26, 37-39]:

$$
\theta_{D}^{3}-\theta_{1 D}^{3} \cong c_{T} C a=c_{T} \frac{\eta v}{\gamma_{\mathrm{lv}}}
$$


where $c_{T}$ is a constant. Hydrodynamic analyses that presume the main force retarding spreading is the viscous impedance utilize such relations as boundary conditions, although uncertainty persists regarding the proper flow conditions near the junction where the dissipation largely occurs (Fig. 4a) [26, 33, 34].

Microscopic analyses attribute the mechanisms controlling spreading to atomic dynamics at the triple line. Several mechanisms have been proposed including: adsorption-desorption, surface diffusion, evaporation-condensation, molecular reorientation and viscoelastic deformation [32]. Using an adsorption-desorption mechanism (depicted in Fig. 4b) in which molecules of the advancing fluid displace those of the receding one at the adsorption sites on the solid surface (Fig. 4), Blake and Haynes [32, 35] developed an expression that can be used to describe the behavior of many low-temperature systems:

$$
\begin{aligned}
C a & =\frac{2 \kappa_{W}^{0} \lambda \eta}{\gamma_{l v}} \sinh \left[\frac{\gamma_{l v}\left(\cos \theta_{1 D}-\cos \theta_{D}\right)}{2 n k T}\right] \\
\kappa_{W}^{0} & =\left(\frac{k T}{h}\right) e^{\frac{-\Delta G_{W}}{N k T}}
\end{aligned}
$$

where $n$ is the number of adsorption sites per substrate area, $\lambda$ their average spacing, $k$ Boltzmann's constant, $T$ temperature, and $h$ Plank's constant. The activation free energy for wetting, $\Delta G_{W}$, derives mainly from the solid-fluid interaction. Most simply, this is the energy barrier to the process required to replace one molecule of the receding fluid from the attachment site on the solid with the one from the advancing fluid.

For many low temperature systems, observed velocities for low viscosity liquids exceed that for glycerol-water in Fig 2, (e.g., Kistler [26] and Refs. therein). A synthesis of the viscous drag and molecular mechanisms to yield a unique $v-\theta$ relationship, as displayed in Fig. 5, has shown some promise for describing spreading on flat, chemically homogeneous substrates [40]. Molecular mechanisms are often controlling at lower velocities owing to the strongly nonlinear $v-\theta$ relationship. This can account for apparent hysteresis implied from higher-velocity data controlled by viscous drag.

From this it should be expected that spreading of liquid metals in regime I should also obey such a unique relationship unless other reactions intervene. Because liquid metals typically have surface energies that are one to two orders of magnitude above those of organic liquids with similar viscosity, the hydrodynamic analysis predicts that for a similar driving force $\left(\theta_{D}^{3}-\theta_{1 D}^{3}\right)$, metals should spread faster. The few dynamic data for high temperature systems do not affirm this although the highest velocities are similar [23, 24] (Fig. 2). If the liquid-substrate chemical interactions are stronger (or more complex see \$2.2) in high-temperature systems than for lowtemperature materials, higher wetting activation energy could be expected yielding slower spreading for similar driving forces, especially for receding fronts but also to a degree for advancing liquids. Thus, the microscopic mechanisms may be limiting over a wider range of $\left(\theta_{D}\right.$ $\left.-\theta_{I D}\right)$ than for simple low-temperature liquids.

\subsection{Adsorption Control}

Equilibrium in multicomponent systems requires that interfaces develop an equilibrium level of adsorption by diffusion among all the phases. The Gibbs adsorption isotherm [41]: 


$$
\mathrm{d} \gamma=-k T \sum \Gamma_{i} \mathrm{~d} \ell \mathrm{n} a_{i}
$$

describes the effects of adsorption on equilibrium interfacial energies. The $\Gamma_{i}$ are the excess amounts of each species that are present per unit area of interface. The equilibrium levels are dictated by the activities, $a_{i}$, of the components in the environment and appear or disappear reversibly with creation or loss of interfacial area. The active metals commonly added to improve wetting on refractory ceramics usually have high surface energies. Thus, it is hard to see how an alloy containing a low fraction of such an element could bring $\theta$ far below $90^{\circ}$ without strong adsorption at the metal-oxide interface. Adsorption may also occur at the other interfaces (Fig. 6) and is relevant both for unreacted interfaces and those with reaction phases. (Attaining $\gamma_{s l}<\gamma_{s v}$ is mandatory; whereas reducing $\gamma_{l v}$ only moves $\theta$ away from $90^{\circ}$, as indicated by equation (1)).

A parallel can be traced between the effects of adsorption with reactive elements and the mutual adsorption expected for metal/oxide systems. (Similar trends should be expected for nitrides and carbides). In these systems, a range of oxygen partial pressures exists in which both phases are compatible and no bulk reaction occurs between them. However, adsorption can occur at all the interfaces, particularly at oxygen activities close to the high and low $p\left(\mathrm{O}_{2}\right)$ ends of the compatibility range [15].

In a proposed model, these adsorption reactions are treated as energetic precursors of bulk reactions [15], because adsorption reactions are often similar to bulk reactions and occur at lower activity. The reactive element, $\mathrm{R}$, could take $\mathrm{O}_{2}$ preferentially from the atmosphere, yielding adsorption of $\mathrm{R}_{\mathrm{y}} \mathrm{O}_{2}$ complexes (e.g., $\mathrm{TiO}$ ) at the different interfaces and at oxygen activities below that for the bulk oxidation of the metal. Two reactions can be compared:

$$
\begin{array}{ll}
\mathrm{xR}_{\{1\}}+\mathrm{O}_{2\{\mathrm{v}\}} \rightarrow \mathrm{R}_{\mathrm{x}} \mathrm{O}_{2\{1\}} & \Delta G_{o x}^{R} \\
\mathrm{yR}_{\{1\}}+\mathrm{O}_{2\{\mathrm{v}\}} \rightarrow \mathrm{R}_{\mathrm{y}} \mathrm{O}_{2\{\mathrm{IF}-\mathrm{i}\}} & \Delta G_{o x-1}^{R}+\Delta G_{a d}^{R-i}
\end{array}
$$

where $\Delta G_{o x}^{R}$ is the free energy for bulk metal oxidation and $\Delta G_{a d}^{R-i}$ is the free energy of adsorption at the i-interface. This adsorption energy differs for the $i$ interfaces. Upon adsorption, such a molecule could partially dissociate, especially at low coverage. The corresponding equilibrium $p\left(\mathrm{O}_{2}\right)$ for the reactions in equations (6) and (7) are:

$$
\begin{aligned}
& p\left(\mathrm{O}_{2}\right)^{\circ} \propto \exp \left[-\frac{\Delta G_{o x}^{R}}{k T}\right] \\
& p_{i}{ }^{*} \propto \exp \left[-\frac{\left(\Delta G_{o x-1}^{R}+\Delta G_{a d}^{R-i}\right)}{k T}\right]
\end{aligned}
$$

Using this definition of the energy to absorb species from the reaction compound rather than from solution, equation (7), enables a direct comparison of the preference for adsorption versus reaction. A characteristic activity (i.e., $p_{i}^{*}$ ) for adsorption is that at which $1 / 2$ coverage of available surface sites occurs. Using the Langmuir adsorption isotherm, permits ready comparison of this with the activity for bulk oxidation, [15]. For simplicity, if the oxide in equation (7) is the lowest valence, with stable oxide of $R$, then $y=x$ and 


$$
\frac{p\left(\mathrm{O}_{2}\right)^{\circ}}{p_{i}{ }^{*}} \propto \exp \left[-\frac{\Delta G_{a d}^{R-i}}{k T}\right]
$$

This parameter dictates the potential for adsorption driven processes. For example, both $\mathrm{S}$ and $\mathrm{O}$ adsorb readily on liquid $\mathrm{Fe}$, but $\mathrm{S}$ does so at a far smaller concentration relative to the solubility limit, which permits much greater reductions in the surface tension. In contrast, $\mathrm{C}$ does not adsorb before forming a carbide [42]. At very low $p\left(\mathrm{O}_{2}\right)$, analogous reduction type adsorption reactions can dominate [15] rather than the oxidation type described in equations (6-10).

Adsorption of $\mathrm{R}_{\mathrm{y}} \mathrm{O}_{2}$ complexes at all the interfaces seems likely, as furnace atmospheres probably exceed $p_{i}{ }^{*}$ and even $p\left(\mathrm{O}_{2}\right)^{\circ}$ for such elements, even in dilute solution. However, in a melt with a Ti concentration exceeding the $\mathrm{O}$ concentration, the effective $p\left(\mathrm{O}_{2}\right)$ is far lower than that in virtually any furnace atmosphere. Hence, the actual adsorption reactions operating may be more complex than are now understood. The effects on wetting undoubtedly depend upon $p\left(\mathrm{O}_{2}\right)$, but the pertinent local oxygen activities may be difficult to ascertain.

In addition, the bulk reaction may occur as

$$
\mathrm{yR}_{\{1\}}+\mathrm{A}_{\mathrm{Z}} \mathrm{O}_{2}=\mathrm{R}_{\mathrm{y}} \mathrm{O}_{2}+\mathrm{zA}_{\{1\}}
$$

and adsorption could involve a complex with one or both products.

Elements that strongly enhance wetting in metal-oxide systems typically can react with several types of compounds (e.g., metallic and ionic) and are often multivalent. Thus, they may seem likely to adsorb at interfaces, being simultaneously bonded to two differing condensed phases. In addition, RO complexes could favorably adsorb, as in equation (7), on either the oxide surface or liquid.

In terms of continuum mechanics, the addition of a reactive element will affect spreading kinetics to the extent that it alters the viscosity and the driving force, $\left(\gamma_{l v}\left(\cos \theta_{I D}-\cos \theta\right)\right)$. For small additions, the effect on $\eta$ should be limited, and the hydrodynamic theory does not predict a strong variation of spreading velocities for a given $\left(\theta-\theta_{I D}\right)$ to result from addition of reactive elements and adsorption. In the microscopic analysis, the relative strength of the reactive element-substrate interaction contains both the important parameters. If adsorption of the reactive element is the kinetically limiting step and its interaction with the substrate is substantially stronger than that of the solvent, the consequent increase in activation energy, $\Delta G_{W}$, will substantially decrease the dewetting velocity of receding fronts. For a given $\left|\theta-\theta_{1 D}\right|$, such big decreases in advancing drop velocities are not expected. Thus, a strong reactive elementsubstrate interaction can result in a much higher driving force and a relatively smaller activation energy and so faster advancing than receding speeds. Many intermediate situations can exist for example, when adsorbing a multicomponent complex involves cooperative motion or steric constraints or when attachment is limited by ledge motion (to expedite reconstruction). Equation (3) is based upon the critical step being adsorption-desorption at the triple line. It does not account for extra time to transport adsorbate from the liquid onto the interfaces, which could be an issue for rapid spreading or for very dilute solutions.

The equilibrium interfacial tensions only describe actual forces when spreading is so slow that the new interfaces form with the direct aid of the adsorbate. If rapid spreading precludes this, several nonequilibrium situations have been hypothesized [2, 29, 33, 43]. One likely situation arises when the primary source of adsorbate for the solid surface is the liquid itself (solvent or solute), but spreading is too rapid for equilibrium levels to actually develop on the 
surface ahead of the liquid [33,43]. A limiting situation is when the surface directly ahead is essentially clean, termed dry spreading (Fig. 6) [33]. Substituting the energy for a pure surface, $\gamma_{s v}^{o}\left(\gamma_{s v}^{o} \geq \gamma_{s v}\right)$, into equation (1), in conjunction with equilibrium interface and liquid energies diminished by adsorption, would often imply low, even vanishing values of the angle $\theta_{1 D}^{d r y}$. Deliberately varying the degree of evaporation of adsorbate from the surface ahead of the junction can yield situations where a front exhibits stable contact angles between those for equilibrium, $\theta_{1 D}$, and the dry spreading limit, $\theta_{1 D}^{d r y}$ [43]. So, for a drop spreading upon an initially clean solid, the effective pull of the solid would thus be intermediate between those described by the two values of the solid surface energy. (Some further increase in driving force for high velocity advancing may also derive from metastable values of $\gamma_{s l}$ being low [43].) This could be the cause for the persistent migration of Pt or Pd "sessile" drops that can be observed on sapphire in open but not closed systems [43], parallel to the "camphor dance" of organics on clean water $[44,45]$. It may also explain why, when a liquid metal is introduced onto a solid of another pure metal (rather than using a pre-equilibrated solid), extensive spreading may ensue toward $\theta$ values well below those for equilibrium [46]. In contrast, a receding drop would tend to leave the adsorbate already established under the liquid. Leaving too much adsorbate would effectively increase the solid surface energy, inhibiting retraction.

To assist comparisons, the critical velocity above which adsorption at the triple line could not accompany and influence the driving force via $\gamma_{s l}$ can be estimated as:

$$
v_{c a} \leq \frac{6 \alpha X_{R} D_{l i q}}{a}
$$

where $D_{l i q}$ is the diffusivity in the liquid, $a$ the atom spacing for the solid, $X_{R}$ the adsorbate fraction in the liquid, and $\alpha(<1)$ a sticking coefficient that describes the chances that an atom jumping from the liquid would attach to the surface to the triple tine. This invokes the minimum barrier consistent with equation 3 and consequently describes an upper bound to $v_{c a}$. Much lower velocities would be needed to permit spreading of adsorbate onto the surface ahead. This would also be lower were $\Delta G_{W}$ much larger than for an atom jump in liquid as would be needed for a receding liquid to remove adsorbate.

Adsorption is responsible for the large reductions of contact angle achieved in several metal/ceramic systems. It achieves these large reductions by adding small quantities of a reactive element to the liquid without forming a reaction product at the interface. This has been discounted in the past for noble-metal/ceramic systems owing to the subsequent reactivity typically seen with $\mathrm{Ti}$ or $\mathrm{Zr}$ additions. However, it is clear that adsorption reactions involving $\mathrm{O}$ promote lower contact angles for $\mathrm{Ni}$ or $\mathrm{Cu}$ on $\mathrm{Al}_{2} \mathrm{O}_{3}$ [[15,47] and Refs. therein]. More recently, it has been realized that at low $p\left(\mathrm{O}_{2}\right)$, adsorption of $\mathrm{Al}$ rich species similarly lower the contact angles in these systems to near or below $90^{\circ}[15,48,49]$. Recent work indicates that even lower contact angles can result from adsorption for $\mathrm{Sn}-\mathrm{Ti}$ and $\mathrm{Cu}-\mathrm{Nb}$ alloys on $\mathrm{Al}_{2} \mathrm{O}_{3}$ [20]. The latter system is the least ambiguous as no interfacial reaction product is found even after cooling. In addition, recent $a b$ initio atomistic computations have shown that submonolayer Ti additions enhance the work of adhesion of such systems (e.g., $\mathrm{Ag} / \mathrm{MgAl}_{2} \mathrm{O}_{4}[50]$ ).

Furthermore, a Cu-Nb liquid ( $1.4 \mathrm{at} \% \mathrm{Nb}$, just above the binary solubility limit) on $\mathrm{Al}_{2} \mathrm{O}_{3}$ exhibits remarkable variability. The contact angles change very slowly, perhaps as $\mathrm{Nb}$ dissolves or the oxygen activity adjusts. In this instance, the fronts for advancing and receding drops 
actually diverge (Fig. 7). ${ }^{2}$ No reaction product is expected, so the spreading to $\theta$ as low as $20^{\circ}$ is considered to be adsorption driven. An element of dry spreading is indicated by the variability. Both the interface and oxide surface energies could be reduced by $\mathrm{Nb}$ containing species $\left(\mathrm{NbO}_{\mathrm{z}}\right)$, so the equilibrium $\theta_{1 D}$ is actually still obtuse. However, if spreading is so fast that the adsorbate cannot reach the solid surface, the advancing front could experience the benefit of a reduced interfacial energy, but not the adverse effect of a lower solid surface energy.

\subsection{Ridge Formation}

In many low-temperature systems (e.g., organic liquids on most ceramics or metals) the vertical component of the surface tension induced force at the triple junction is resisted by elastic distortions of the solid. It is then accurate to describe the substrate as ideally rigid and insoluble. However, for molten metals or oxides on ceramics or metals, exposure temperatures are typically $\geq(0.2-0.5) T_{m}{ }^{s}\left(T_{m}{ }^{s}\right.$ being the substrate melting point), which will allow some local diffusion or solution/precipitation. Then, attaining equilibrium at the junction requires motion of the triple line both horizontally and vertically [51-53] (two-dimensional, 2-D case) which leads to two independent relations:

$$
\frac{\sin \phi_{s}}{\gamma_{l v}}=\frac{\sin \phi_{l}}{\gamma_{s v}}=\frac{\sin \phi_{v}}{\gamma_{s l}}
$$

where $\phi_{s}, \phi_{l}$ and $\phi_{v}$ are the equilibrium dihedral angles in the solid, liquid and vapor phases, respectively. Under such conditions, at a certain point a small ridge will develop at the triple junction by atomic migration near the contact line. The triple junction will remain attached to the ridge unless a sudden perturbation drives the macroscopic contact angle outside a stability range that depends on the ridge orientation, causing the wetting front to break away $[52,54]$. The ridge will evolve and propagate until complete equilibrium is attained. Equilibrium involves constant chemical potential shapes (i.e., constant curvature for isotropic systems) [41]. Although attaining full equilibrium may take an impossibly long time, the small ridge can be highly influential.

Recently, it has been recognized that triple line ridges can often control spreading rates and hysteresis for high-temperature systems [52]. A parallel between triple line ridging and the Mullins theory for grain boundary grooving $[55,56]$ has been established. Essential aspects of small-ridge behavior have been revealed for the situation where interfacial diffusion is rate controlling [52]. A time scale exists in which a ridge is very small compared with the radius of curvature for the liquid, $h / R<<0.1$, and can be carried by a moving front. In this regime, which is defined as regime II spreading, the capillary forces drive the macroscopic $\theta$ toward a value very near that satisfying Young's equation, with a driving force approximated by that for regime $\mathrm{I}\left(\gamma_{l v}\left(\cos \theta_{l D}-\cos \theta\right)\right)$ but with spreading kinetics dictated by the rate at which the attached ridge moves [52].

In general, the relative rates of ridge growth, $v_{\perp}$, and spreading, $v_{/ /}$, vary in time and depend upon controlling kinetics. Two steady-state conditions (corresponding to advancing and receding fronts) exist in which a liquid front can move with constant velocity attached to a ridge of constant height (i.e., $\left.v_{\perp}=0\right)[52,56]$. For macroscospic contact angles in the range delimited by the associated angles $\theta_{s s}{ }^{r e c}$ and $\theta_{s s}{ }^{a d v}$, the liquid front will remain attached to a ridge and

\footnotetext{
${ }^{2}$ The data here and in Fig. 13 were taken following procedures described in Refs. [10, 11].
} 
decelerate, while the ridge grows faster as the angle sweeps toward $\theta_{1 D}$. From the exact solutions, it can be infered that the transient velocities will vary as:

$$
\begin{array}{ll}
v_{/ /}=\frac{\omega D_{s l} \gamma_{s l} \Omega}{k T h^{3}} f_{r}\left(\theta, \frac{\gamma_{l v}}{\gamma_{s l}}\right)\left(\cos \theta_{1 D}-\cos \theta\right) & \theta_{s s}^{r e c}<\theta<\theta_{l D} \\
v_{/ /}=\frac{\omega D_{s l} \gamma_{s v} \Omega}{k T h^{3}} f_{r}\left(\theta, \frac{\gamma_{l v}}{\gamma_{s v}}\right)\left(\cos \theta_{1 D}-\cos \theta\right) & \theta_{l D}<\theta<\theta_{s s}{ }^{a d v}
\end{array}
$$

with $\omega D_{s i}$ being the width-diffusivity product for the specific interface, $\Omega$ the atomic volume, and $h$ the ridge height [52]. A junction moving on a smooth surface should form a ridge spontaneously by diffusion once $\theta$ is within the range delimited by the steady-state solutions, and sharp perturbations (such as scratches) may act as incipient ridges. In addition, as $\left|\theta-\theta_{1 D}\right|$ diminishes, the front should be increasingly resistant to break away triggered by a disturbance or change in liquid volume. In contrast, when $\theta$ is outside the growth range, any ridge attached to a junction would shrink during spreading, leading to a diminishing drag force. Hence, such a liquid spreading on a smooth surface would have no tendency to form a ridge and would resist being pinned upon passing a ridge-like asperity. The system would also be ever more susceptible to break away at higher $\left|\theta-\theta_{1 D}\right|$.

For a drop spreading stably in regime II, the extent of ridge growth should depend on the drop size and change in angle (i.e., $\Delta h \propto r\left[\mathrm{~F}\left(\theta_{f}\right)-\mathrm{F}\left(\theta_{i}\right)\right] \propto \Delta r$, independent of temperature). The velocities scale with the controlling diffusivity, leading to faster spreading and ridge growth at higher temperature [52]. However, a condition exists wherein a liquid can spread on a flat surface quickly enough to suppress initiation of ridge growth (even if $\theta_{s s}{ }^{r e c}<\theta<\theta_{s s}{ }^{a d v}$ ). Here diffusional fluxes are limited by the actual atomic jump frequency rather than being virtually infinite as $h \rightarrow 0$. There should be a spreading velocity beyond which ridge formation is impossible, because the jump frequency becomes too slow compared to the spreading velocity and the first "atomic ridge" cannot form at the triple junction. This critical velocity, $v_{c r}$, can be estimated as:

$$
v_{c a}=\frac{6 D_{s l}}{a}
$$

where $a$ is a jump distance and $D_{s l}$ the surface diffusion coefficient at the solid/liquid interface. Thus, in the transitional temperature regime, a fast front (in regime I) could sweep into the ridgegrowth range until the driving force $\left(\theta-\theta_{1 D}\right)$ is diminished enough that the slow spreading allows the initiation of ridge formation. The requisite distance traveled before ridge initiation would shrink, and the extent of ridge growth while approaching $\theta_{1 D}$ would increase with rising temperature. This trend is implied by observations that spreading times are longer at higher temperatures for various $\mathrm{Cu}-\mathrm{Ag}$ alloys on mullite [11].

Ridges have been found in several systems. They span heights from $10 \mathrm{~nm}$ for $\mathrm{Cu}$ on $\mathrm{Al}_{2} \mathrm{O}_{3}$ (Fig. 9), to $0.2 \mu \mathrm{m}$ for $\mathrm{Ni}$ on $\mathrm{Al}_{2} \mathrm{O}_{3}$, to $70 \mu \mathrm{m}$ for silicate liquids on $\mathrm{Co}$ at $99 \%$ of $T_{m}$ for the Co [52]. The shape of ridges with sizes up to $\sim 1 \mu \mathrm{m}$ indicates that the kinetics are dictated by diffusion at the interface or within the liquid [52], the same mechanisms that control the growth of grain boundary grooves at the solid/liquid and solid/vapor interfaces [57]. For liquid Cu drops on sapphire, advancing and receding fronts approach a common angle after many minutes (involving $v \sim 10^{-6} \mathrm{~m} / \mathrm{s}$ which is an indication that the ridge is small and that spreading occurs 
under regime II conditions. Thus, the final angle is held to satisfy equation (1). A recent work suggests that at temperatures $1100-1500^{\circ} \mathrm{C}, \omega D_{s l} \approx \omega D_{s v} \approx 10^{-14}-10^{-18} \mathrm{~m}^{3} / \mathrm{s}$ for most noble metals on alumina [57]. The corresponding upper limit for the velocity of a $10 \mathrm{~nm}$ ridge is $10^{-}$ ${ }^{6}-10^{-10} \mathrm{~m} / \mathrm{s}$ based on the steady state solutions [52]. This velocity is far slower than typical spreading velocities for organic liquids $[26,32]$, but similar to or even slower than those recorded in many high-temperature systems (Fig. 2). Apparently, most of the observable spreading occurs with ridges of $h<10 \mathrm{~nm}$, and most of the ridge growth ensues when the front is virtually stationary. ${ }^{3}$

Thus, the dynamic velocity of high temperature liquids should behave essentially as sketched in Fig. 8. For high values of $\left|\theta-\theta_{1 D}\right|$, spreading will be in regime I, with a unique $v(\theta)$ as discussed in $\$ 2.1$ and $\S 2.2$ (Fig.5). Within the interval for ridge-controlled spreading, there is no unique wetting velocity for each dynamic contact angle, but rather a broad range of velocities that depend on the ridge height (Fig. 8). That height, in turn, depends on the size of the perturbation that originated it and the contact angle at which it actually initiated. This could be one source of the wide variability in spreading velocities recorded in high-temperature systems for similar liquids. The spreading in regime I can be far faster than in regime II in reactive systems if the adsorption step can be quick enough. For an advancing front $v_{c a} / v_{c r}=\alpha X_{R} D$ liq/D $D_{s l}$ from equations (12) and (15). For the range of $\omega D_{s l}$ quoted just above, and taking $\omega=1 \mathrm{~nm}$, and $D_{\text {liq }}=10^{-9} \mathrm{~m}^{2} / \mathrm{s}, D_{\text {liq }} / D_{s l} \sim 10^{2}-10^{-2}$, with increasing temperature. Hence, rapid regime I type spreading with adsorption would be likely at more moderate substrate temperatures even for dilute adsorbates, unless $\alpha$ is very small ( $\Delta G_{W}$ is larger than the minimum). This seems consistent with a recent observation of extremely fast spreading to low angles with Ni-Pd-Ti on vitreous carbon $(\mathrm{Cv})$ [23] (The dynamic velocities slightly exceed those shown in Fig.2 for $\mathrm{Ni} / \mathrm{SiC})$.

\subsection{Reaction Phase Formation}

Frequently, a supersaturated species from a liquid can react with a solid to form a new phase more or less continuously along the original solid/liquid interface. Although the presence of a reaction product would undoubtedly affect the spreading rates and ultimate contact angle, such phases are typically seen postmortem, without knowing when they formed with respect to spreading. Several distinct situations may exist (Figs. 3 and 9), depending upon the rates of nucleation and continued reaction, which would sometimes be far slower than for the liquid to flow, even with concurrent ridging [43].

With slow nucleation, such a spreading fluid can leave behind a metastable interface with well-defined properties, which may include low $\gamma_{s l}$ owing to adsorption of reactant (Fig. 3a). The rapid fluid flow must accommodate adsorption to actually reduce $\gamma_{s l}$ just behind the triple junction. This could yield a transient situation (with potential for later liquid retraction), wherein a very low $\gamma_{s l}$ develops and is followed by an increase of $\gamma_{s l}$ as growth of the reaction phase at places along the interface diminishes the supersaturation of a chemically active species, $R$.

If a new compound can nucleate sufficiently rapidly, several other configurations might be possible (Fig. 10). In one, the liquid wets to the edge of the reaction layer, but not onto the solid ahead (Figs. 3c and 10). The reaction layer and liquid could then extend together, as limited by reaction kinetics, and the apparent contact angle of the drop would decrease. Limited by counter-diffusion of reactants across the tip of the reaction layer, spreading would be

\footnotetext{
${ }^{3}$ For the larger ridges, growth is predominantly by diffusion within the liquid [57].
} 
comparatively slow for more than nanometer-thick layers. Alternatively, the compound may extend beyond the liquid front (Fig. 10). The drop would then seek a shape dictated by surface and interfacial $\gamma$ 's for the reaction product. A system could switch from the first to the second of these as the liquid depletes or the reaction product extends, with further reaction becoming ever slower if limited by diffusion. A frequently observed variant occurs, however, where the reaction product is porous and contains an interconnected liquid, through which the reactant can move rapidly $[11,43,58]$.

A major issue is, therefore, whether the new phase can form during the time it takes for the liquid front to spread. A limiting situation is attained if the liquid spreads so quickly, exceeding a critical velocity, that not enough time exists to even nucleate the reaction product at the substrate near the spreading front. The steady-state frequency for nucleating a new phase, $\beta$, from the liquid at the substrate-liquid interface can be approximated as $[59,60]$ :

$$
\begin{aligned}
& I_{s}=N_{s} 6\left(\frac{D^{*}}{a^{2}}\right) \exp \left[-\frac{16 \pi \gamma_{\beta l}^{3}}{3\left(\Delta G_{v}\right)^{2} k T} f\left(\theta_{1}\right)\right] \\
& f\left(\theta_{1}\right)=\frac{\left(2+\cos \theta_{1}\right)\left(1-\cos \theta_{1}\right)^{2}}{4}
\end{aligned}
$$

where $\gamma_{\beta l}$ is the interfacial energy between $\beta$ and the liquid, $\Delta G_{v}$ the free energy change driving transformation, $D^{*}$ the local diffusivity controlling attachment to an embryo, and $N_{s}$ the areal site density for nuclei in the system. The factor $\mathrm{f}\left(\theta_{l}\right)$ describes the reduction in nucleus energy if the new phase partially wets the interface, where the wetting angle of the new $\beta$ phase on the interface, $\theta_{l}$, (Fig. 11) is a key determinant. If $\theta_{l} \rightarrow 0$, there is no barrier to nucleation. The critical velocity can be roughly estimated as $a I_{s}$, the time to pass a site so fast that there is just a chance of nucleating on it. Taking $I_{s}=1$, then

$$
v_{c n}=6\left(\frac{D^{*}}{a}\right) \exp \left[-\frac{16 \pi \gamma_{\beta l}^{3}}{3\left(\Delta G_{v}\right)^{2} k T} f\left(\theta_{1}\right)\right]
$$

This overestimates by factor $<a^{2} / \mathrm{A}$, where $\mathrm{A}$ is the area of interface where spreading occurs. However, for rapid spreading, a further adjustment for the slower transient nucleation rates would be compensating.

In cases of interest, adsorption at the original interface would lower $\gamma_{s l}$ and promote wetting of the liquid onto the substrate. The extent of further adsorption as the activity of $\mathrm{R}\left(a_{R}\right)$ increases to and beyond the equilibrium level to form a new phase (as sketched in Fig. 11) is germane. In the extreme case, with $\theta_{l} \rightarrow 0$, upon approaching the solubility limit, there is a smooth transition, energetically, from monolayer adsorption through some layers of multilayer adsorption to a reaction product that fully wets the original interface(c). In contrast, where only monolayer adsorption is favorable (a), some supersaturation of $\mathrm{R}$ can occur without nucleation, as the new phase does not fully wet the interface. A longer time will be required; in effect, a lower sticking probability applies for a reactive element attachment to form the nucleus than to form the adsorbate $\left(v_{c n}<<<v_{c a}\right)$. In this instance, the metastable interfacial energy can decrease to very low levels (Fig. 11), giving much lower wetting angles, $\theta$, for the liquid on the substrate than would apply after depletion of $\mathrm{R}$ back to the solubility limit. In an intermediate situation (b), multilayer adsorption initiates at a concentration above the solubility limit; at a slightly higher, critical concentration, $X_{R}^{\dagger}$, the heterogeneous nucleation barrier would virtually vanish. 
(Note that the partial wetting condition, $\theta_{1}>0$, alludes to isolated particles of reaction product, not to the polycrystalline layer that can eventually cover the interface, metastably, during extensive reaction.)

Extensive theory for the extent and type of adsorption prior to new phase formation has been developed for fluids on undeformable solids and shown to depend strongly upon the relative strength of the substrate-adsorbate and adsorbate-adsorbate interactions [61-63]. With a strong substrate interaction, complete wetting by the new phase is expected. A modified treatment of nucleation of a nearly pure phase during solidification from an adsorption perspective has implied somewhat similar expectations - that is the barrier vanishes at a critical undercooling for a coherent nucleus and the degree of undercooling varies greatly with changes in the atomic interaction energies [64]. Thus, the critical speed for allowing this barrierless reaction to occur at a spreading front may simply be a multiple of that for adsorbing a single layer, equation (12). (Although the driving force is relatively insensitive to thickness, a kinetic transition to diffusion control would require much longer times to develop thicker layers.)

However, for most solids forming on solids, it is unclear that complete wetting will occur and be maintained if the interface breaks coherency; that is, the strain misfit will drive island formation. Then, in the limit of extremely fast nucleation forming discrete particles at the spreading front, the resultant roughness may, in fact, pin and slow the spreading front. Other complexities arise for compound formation in that having a continuous transition from adsorption to second phase formation would imply that the multilayer adsorbate would be of mixed composition. This, in turn, would imply a wider family of interaction energies, which in some cases would favor single layer adsorption much more than multilayer (e.g., stronger inplane than out-of-plane adsorbate-adsorbate interactions). In addition, when reaction requires dissolution of some substrate material (equation 11), the times for multilayer adsorption and nucleation of second phase could vastly exceed that needed to form the first adsorption layer.

Additions of $\mathrm{Ti}$ to $\mathrm{Cu}$ and $\mathrm{Cu}-\mathrm{Ag}$ eutectic liquids enhance wetting on oxides. In many cases, reaction products form extensively near the interface; the details depend upon the Ti level [10]. For higher Ti levels, the multiphase reaction morphology is complex [10]; most or all of it probably forms on solidification. The angles vary notably, even for similar samples; with 2 at $\%$ $\mathrm{Ti}$, angles on various samples of sapphire and commercial $\mathrm{Al}_{2} \mathrm{O}_{3}(99.8$ and $96 \%)$ spanned $\theta \sim$ 20-40 (Fig. 2) [19].

Cross-sectional analysis near the edge is instructive. After two hours, the $2 \%$ samples exhibit a layer of $\mathrm{TiO}$ thick enough to account for most of the Ti (Fig. 12). Typically, a uniform layer extends to the edge of the contact (Fig. 12), a geometry seemingly attributable to the reaction and liquid spreading together. However, if that were the case, the spreading rates that correspond to the concurrent growth rate of the reaction product would be much slower than observed. In contrast, note the sample in which the front had arrested at a higher angle is also shown in Fig. 12. Here, near the edge of the contact, the reaction product is very much thinner. The proposed mechanism, depicted in Fig. 3, is that of the liquid actually spreading ahead of the reaction front, until a triple line ridge becomes big enough to essentially arrest spreading at $\theta>$ $\theta_{I D}$. Subsequently, reaction product will form rather uniformly under the drop. If the arrested macroscopic angle is large enough and the front is dislodged from this position, some driving force for further advance still remains despite the depletion of reactive element. Hence, the front will move ahead until, again, it is virtually re-arrested by a growing ridge. The occurrence of such arrest and jump-off may depend on heterogeneities which trigger premature arrest 
stochastically in limited ranges of composition. Similar behavior has also been observed for Ag$\mathrm{Cu}$ liquids with low levels of $\mathrm{Zr}$ on mullite [43]. In both instances, at higher $\mathrm{R}$ contents, the contact angles attained were nearer zero, and evidence of such jump-off after arrest was not obvious.

In a highly reactive system such as $\mathrm{Al}$ on dense mullite, $\mathrm{SiO}_{2}$, or kaolinite in which the aluminum reduces the $\mathrm{SiO}_{2}$ [58], a porous reaction product forms and soon extends far beyond the liquid, leaving a drop with $\theta \sim 60-90^{\circ}$. Spreading to very low angles is never observed [58, 65], in contrast to expectations from the models that require high chemical reactivity to promote spreading $[28,29]$. This may imply that isolated nucleation at the growth front actually stalls the spreading, rather than a continuous layer of smoother, barrier free reaction phase forming concomitantly and driving very rapid spreading.

\section{DISCUSSION}

Reactive spreading involves several, sometimes competitive, processes, with differences among them dictating evolution along different paths. We divide the reactive wetting process into a series of stages: liquid flow, adsorption of reactive element, ridging, nucleation, and growth of the reaction phase (Fig. 3). The objective is to determine the structure and composition of the triple junction (Fig. 10) for each stage to identify what drives the decrease of contact angle as well as which process controls the spreading kinetics.

Several observations affirm that spreading to low contact angles can be driven by adsorption at the interface either without, or prior to, forming a reaction phase. Evidently, having a large relative interfacial adsorption energy for an RO complex, $-\Delta G_{a d}{ }^{R-s l}$ (equation 7), can yield good wetting at contents below the solubility limit (that for reaction or precipitation). In contrast, marginal levels of $-\Delta G_{a d}{ }^{R-s l}$ can promote good wetting only with supersaturated liquids. The primary question is whether adsorption drives spreading prior to forming a new phase or whether the phase forms first and spreads with, or even ahead of, the front. A second concern is what controls rates and arrests spreading. For adsorbates with an associated low activation energy for wetting, $\Delta G_{W}$, spreading velocities are similar to those for spreading of pure liquid. Consequently, at temperatures well below $T_{m}{ }^{s}$, where the interface diffusivity is low, a simple adsorption reaction could occur far quicker than the critical time to induce a ridge. Thus, for a wide temperature range, a front that has not attached to a ridge could move essentially in regime I but with adsorption at the interface. Such fast spreading has been observed in several reactive systems using the drop transfer method (Fig. 2) [23].

Comparing equations (12) and (17) shows that the highest velocity that would allow nucleation at the spreading front is reduced from the adsorption velocity, $v_{a}$, primarily by the factor, $\exp \left\{-\left[16 \pi \gamma_{\beta l}{ }^{3} / 3\left(\Delta G_{v}\right)^{2} k T\right] \mathrm{f}\left(\theta_{l}\right)\right\}$. For supersaturations below the critical level to bring $\theta_{l}$ $=0$, this can be orders of magnitude less than unity. Then, a liquid in regime I could spread with a low metastable contact angle, $\theta_{I D}$, because it leaves behind a metastable interface with low solid /liquid interfacial energy. A liquid front spreading at a lower velocity limited by ridge drag (regime II) would easily have time to establish equilibrium adsorbate at the interface. As the velocity drops with larger ridges, there would increasingly be time to permit nucleation at the interface and also adsorption on the surface ahead of the triple line. The reported results indicate that apparently, in many reactive cases, a finite nucleation barrier does exist for forming the reaction product. The large decrease observed in contact angle derives from adsorption, and the spreading rates are dictated by migration or pinning of a triple point ridge. This seems to be the case for the $\mathrm{Ag}-\mathrm{Cu}-\mathrm{Ti} / \mathrm{Al}_{2} \mathrm{O}_{3}$ system (Fig. 2) and also in the spreading of $\mathrm{Cu}-\mathrm{Ti}$ [66] alloys on 
$\mathrm{Al}_{2} \mathrm{O}_{3}$, where there is an appreciable decrease in the contact angle. Reportedly, the reaction product does not completely cover the solid/liquid interface or reach the triple junction [66]. The schematic in Fig. 3 suggests a sequence that is likely to apply, at least to some extent, in many cases.

Uncertainty remains as to the actual equilibrium contact angles in reactive systems and the role of nonequilibrium capillary forces. For example, the divergence of advancing and receding fronts for the $\mathrm{Cu}-\mathrm{Nb} / \mathrm{Al}_{2} \mathrm{O}_{3}$ case strongly implies that dry spreading is operative. The hypothetical sketches in Fig. 6 illustrate plausible adsorption effects on interfacial energies. But these sketches would not imply the strong divergence between equilibrium and dry spreading which simply requires $\gamma_{s v}<\gamma_{s l}$ at all activities of R, $a_{R}$. Finally, it is noted that enhanced spreading has also been attributed to composition gradients very near the front, causing variations in $\gamma_{l v}[67,68]$; this could augment the effects of nonequilibrium surface adsorption, but could not, in themselves, cause the divergence seen with the $\mathrm{Cu}-\mathrm{Nb}$ system (Fig. 7).

Experiments conducted with $\mathrm{Sn}$-Ti alloys on $\mathrm{Al}_{2} \mathrm{O}_{3}$ show a large reduction of contact angles with respect to those of pure tin which is believed to result from adsorption as macroscopic reaction product is not found by SEM at the interface even after cooling [20]. Low angles $(\theta$ $<25^{\circ}$ ) can be attained at $1000^{\circ} \mathrm{C}$ (Fig. 1), using Ti contents well below the binary solubility limit; it is $\sim 30$ at\% at which $\mathrm{Sn}_{6} \mathrm{Ti}_{5}$, is stable. For "receding" specimens, contact fronts first typically retract after the oxide skin reduces, but then advance while the Ti dissolves. Such experiments often involve mechanical mixtures of $\mathrm{Ti}$ in the $\mathrm{Sn}$, and dissolution can take many minutes. If smaller, pre-alloyed pieces of 50Sn50Ti (full symbols, Fig. 13) were used instead of pure Ti, the angles tend to be lower. Considerable wetting hysteresis persists, but apparently the equilibrium angle is acute.

The hysteresis and variability for $\mathrm{Sn}-\mathrm{Ti}$ and $\mathrm{Ag}-\mathrm{Cu}-\mathrm{Ti}$ on $\mathrm{Al}_{2} \mathrm{O}_{3}$ are believed to mainly reflect drag from triple line ridges. Ridging theory indicates that for advancing fronts, ridges should form for dynamic contact angles such that $\theta_{s s}{ }^{a d v}>\theta>\theta_{1 D}$. However, in many systems, $\theta$ often drops seemingly continuously from $\sim 100^{\circ}$ to less than $10^{\circ}$ over some minutes $[7,11,66]$. Such a change is far larger than any difference between static values of $\theta_{s s}{ }^{a d v}$ and $\theta_{1 D}$ [52], yet the velocities are not typical of regime I, compared to those for $\mathrm{Ag}-\mathrm{Cu}-\mathrm{Ti}$ on $\mathrm{Al}_{2} \mathrm{O}_{3}$ and those low-temperature liquids in Fig. 2. The time to melt allows ridges to form and influence the associated adjustments of shape and volume. Apparently, $\theta_{1 D}$ then reduces gradually as the reactive element is released, and its activity in the liquid $\left(a_{R}\right)$ changes continuously. Perhaps when $a_{R}$ is evolving with time, the fronts can travel large distances under ridge drag control, but without much ridge growth, by having $\theta \sim \theta_{S S}{ }^{a d v}\left(a_{R}\right)$ until $\theta_{I D}$ and $\theta_{S S}{ }^{a d v}$ become stable. Then, the front approaches $\theta_{I D}$, only until the ridge becomes so large that velocities become negligible. Alternatively, the scenario may involve several breakaway events. In either event, further study is needed, especially to understand how the largest hysteresis observed $\left(\mathrm{Nb}-\mathrm{Cu} / \mathrm{Al}_{2} \mathrm{O}_{3}\right)$ exceeds expected differences in $\theta_{S S}{ }^{a d v}$ and $\theta_{I D}$ and involves both ridging effects, contributions from dry spreading by causing differences in driving force, and perhaps other effects such as evaporation of adsorbate from surfaces.

The behavior of highly reactive systems, in which the nucleation barrier of the reaction product is low and a compound forms quickly, is a matter of controversy. It has been widely believed that reaction products are better wet than the parent. However, the intrinsic wetting angle on a reaction phase may not be very low if it too is a refractory compound with a high band gap or formation energy $[2,5,6,11,28]$. Mutual adsorption effects would reduce $\theta$, but not 
likely cause exceptionally low angles for an M-R alloy on a layer of refractory $\mathrm{R}_{\mathrm{x}} \mathrm{O}_{2}$. This is in accord with other observations, although such experiments are fraught with errors and artifacts from oxide skin formation and ridging. For example, Ag-In-Zr alloys reportedly exhibit only fair wetting on $\mathrm{ZrO}_{2}\left(\theta \approx 60-100^{\circ}\right)$ [22]; even for $\mathrm{Cu}$-Ti alloys on $\mathrm{TiO}_{1-\mathrm{x}}$, which has a much lower band gap, the reported angle is $72^{\circ}$ [2]. Also, the roughness of a typical polycrystalline reaction layer could inhibit advance. Thus, it is deemed unlikely that extensive spreading occurs on the reaction layer in the case of most oxides. The best way to achieve low $\theta$ may be to have a high enough concentration of active element to give strong adsorption and (metastable) interfaces with low $\gamma_{s l}$, and possibly high $\gamma_{s v}$, but where spreading precedes reaction. Then, subsequent retraction may actually be favored, but inhibited by ridging or roughness. With excessive reactivity, the new compound may too soon extend beyond the spreading front but not carry it, as with $\mathrm{Al} /$ silicates $\left(\theta \approx 60-90^{\circ}\right)$. Also, in some cases the capillary pressure of a liquid infiltrating through a porous reaction product could drive slow spreading within it [58] .

The situation may differ for graphite, diamond or $\mathrm{SiC}$, as metallic carbide reaction products can be readily wet $[6,9]$, but it is complicated. For $\mathrm{Cu}-\mathrm{Cr}$ alloys, which can yield $\theta \sim 10^{\circ}$, there is some early evidence that good wetting on graphite requires critical levels of $\mathrm{Cr}$ that cause the presence of a continuous reaction product that is well wetted by the alloy [9, 69, 70]. A need to actually spread on a reaction phase could indicate that the relative adsorption energy, $\Delta G_{a d}{ }^{R-s l}$, is unfavorable, so that interfacial adsorption does not precede reaction, or that adsorption also lowers $\gamma_{s v}$ such that dry spreading is necessary to attain low angles. The more recent droptransfer studies give speeds that seem too high for concurrent formation of thick reaction products, but the much higher speeds seen with $\mathrm{Ni}-\mathrm{Pd}-\mathrm{Ti}$ [23] than with $\mathrm{Cu}-\mathrm{Cr}$ [24] on vitreous carbon $(\mathrm{Cv})$ require further study for explanation.

This analysis yields insights about the mechanisms necessary for spreading when a system is in a given wetting regime. A critical issue in treating kinetics is to identify relevant driving forces. Those in equations (1-3, 11-15) depend upon interface tensions, as modified by (5). If these are not meaningfully definable or are unstable, then fundamentally new treatments are needed. Moreover, real-life situations are complex, and other factors may influence spreading. Initial heterogeneities and oxide skins formed on multicomponent liquids can markedly influence the ensuing evolution. Exchange with the atmosphere may also be slower than often realized [71]. In addition, temperature gradients have been neglected, with the understanding that heat absorbed during melting or liberated during reactions may not only cause time delays, but also spatial variations in surface tension.

\section{CONCLUDING REMARKS}

Reactive wetting behavior is highly complex. The analysis presented in this paper divides reactive wetting into a series of stages and outlines the basic, mechanistic steps involved in spreading that merit further kinetic study. It also establishes key parameters that dictate the path of wetting and reaction. It is expected that this approach can lead to a better understanding of the phenomena, as well as to improved quantitative models.

The perception persists that good wetting is only attained with concentrations of reactive element high enough to form a reaction product. This is demonstrably not true for some cases. Furthermore, it is proposed that, in many reactive cases, the large decrease observed in wetting angle is a consequence of adsorption, and that frequently observed slow spreading rates and wetting hysteresis are often controlled by the dynamics of a triple line ridge. A result of the analysis is that the degree to which good wetting can occur below the solubility limit versus 
requiring supersaturated liquids depends upon the relative adsorption energies. Extension of adsorption relations into the supersaturation regime is justifiable if a barrier exists to nucleation of the reaction phase. Such a barrier can stablize surfaces or interfaces with very low metastable $\gamma_{s l}$. Obviously, advancement in this field requires investigating equilibrium and nonequilibrium adsorption reactions and related forces (dry spreading) that may further promote spreading.

\section{Acknowledgments}

This work was supported by the Director, Office of Energy Research, Office of Basic Energy Sciences, Materials Sciences Division of the U. S. Department of Energy under Contract No. DE-AC03$76 \mathrm{SF} 00098$. 


\section{REFERENCES}

1. Chatain, D., Rivollet, I., and Eustathopoulos, N., Journal de Chimie Physique et de Physico-Chimie Biologique, 1986, 83, 561.

2. Naidich, Y. V., Progress in Surface and Membrane Sciences, 1981, 14, 353.

3. Kurkjian, C. R. and. Kingery, W. D, J. Phys. Chem., 1956. 60, 961.

4. Humenik, M., Jr., and Kingery, W. D., J. Amer. Ceram. Soc., 1954. 37, 18.

5. Li, J.-G., J. Amer. Ceram. Soc., 1992, 75, 3118.

6. Stoneham, A. M., Duffy, D. M., and Harding, J. H., in Designing Ceramic Interfaces II: Understanding and Tailoring Interfaces for Coating, Composite and Joining Applications, ed. S.D. Peteves, Committee of European Communities: Luxembourg., 1993, p. 573.

7. Nicholas, M. G., Valentine, T. M., and Waite, M. J., J. Mat. Sci., 1980, 15, 2167.

8. Nicholas, M. G., and Mortimer, D. A., Mat. Sci. Tech., 1985, 1, 657.

9. Scott, P. M., Nicholas, M., and Dewar, B., J. Mat. Sci., 1975, 10, 1833.

10. Loehman, R. E., and Tomsia, A. P., Acta Met. Mater., 1992, 40, S75.

11. Loehman, R. E., and Tomsia, A. P., J. Amer. Ceram. Soc., 1994, 77, 271.

12. Eustathopoulos, N., Chatain, D., and Coudurier, L., Mat. Sci. Eng., 1991, A135, 83.

13. Nogi, K., Transactions of the Japanese Welding Research Instititute, 1993, 22, 183.

14. Kritsalis, P., Coudurier, L., and Eustathopoulos, N., J. Mat. Sci., 1991, 26, 3400.

15. Saiz, E., Tomsia, A. P., and Cannon, R. M., in Ceramic Microstructures: Control at the Atomic Level, eds. A. P. Tomsia and A. M. Glaeser, Plenum Press: New York.1998, p. 65.

16. Naidich, Y. V., Zhuravljov, V. S., and Erumina, N. I., J. Mat. Sci., 1990, 25, 1895.

17. Meier, A., Chidambaram, P. R., and Edwards, G. R., J. Mat. Sci., 1995, 30, 3791.

18. Li, J.-G., J. Mater. Sci. Let., 1992, 11, 1551.

19. Tomsia, A. P., in preparation.

20. Tomsia, A. P., Fopianno, S., and Saiz, E., Reactive Wetting and Spreading in Sn$\mathrm{Ti} / \mathrm{Al}_{2} \mathrm{O}_{3}$ and $\mathrm{Cu}-\mathrm{Nb} / \mathrm{Al}_{2} \mathrm{O}_{3}$ Systems, in preparation.

21. Ambrose, J. C., Nicholas, M. G., and Stoneham, A. M., Acta Met. Mat., 1992, 40, 2483.

22. Xue, X. M., Sui, Z. T., and Wang, J. T., J. Mater. Sci. Let., 1992, 11, 1514.

23. Grigorenko, N., Poluyanskaya, V., Eustathopoulos, N. and Naidich, Y., in Interfacial Science of Ceramic Joining, eds. A. Bellosi, T. Kosmac, and A. P. Tomsia, Kluwer Academic Publishers, Dordrecht, The Netherlands, 1998, p. 57.

24. Voitovitch, R., Mortensen, A., Hodaj, F. and Eustathopoulos, N., Acta Mater., 1999, 47, 117.

25. Burley, R. and Kennedy, B .S., Chem. Eng. Sci., 1976, 31, 901.

26. Kistler, S. F., in Wettability, ed. J. C. Berg, Marcel Dekker, New York, 1993, p. 311.

27. Kritsalis, P., Merlin, V., Coudurier, L. and Eustathopoulos, N., Acta Met. Mat., 1992, $40,1167$.

28. Eustathopoulos, N. and Drevet, B., J. Phys. III, 1994, 4, 1865.

29. Aksay, I. A., Hoge, C. E. and Pask, J. A., J. Phys. Chem., 1974,78, 1178.

30. Yost, F. G. and Romig Jr, A. D., in Material Research Society Symposium Proceedings 1988, , 108, p. 385.

31. Adamson, A.W., Physical Chemistry of Surfaces. 4th ed. 1982, New York: John Wiley \& Sons.

32. Blake, T. D., in Wettability, ed. J. C. Berg, Marcel Dekker, New York., 1993, p. 251.

33. de Gennes, P. G., Rev. Mod. Phys., 1985, 57, 827.

34. Davis, S. H., J. Appl. Mech., 1983, 50, 977.

35. Blake, T. D. and Haynes, J. M., J. Coll. Interf. Sci., 1969, 30, 421.

36. Ruckenstein, E. and Dunn, C. S., J. Coll. Interf. Sci., 1977, 59, 135.

37. Hoffman, R. L., J. Coll. Interf. Sci., 1975, 50, 228.

38. Voinov, O. V., Izvestiya Akademii Nauk SSSR, Mekhanika Zhidkosti i Gaza, 1976, 5, 76.

39. Tanner, L. H., J. Phys. D (Appl. Phys.), 1979, 12, 1473.

40. Schneemilch, M., Hayes, R. A., Petrov, J. G. and Ralston, J., Langmuir, 1998, 14,7047.

41. Gibbs, J. W., Collected Works. Vol. I. 1931, New York, Longmans.

42. Halden, F. A. and. Kingery, W. D, J. Phys. Chem., 1955, 59, 557. 
43. Cannon, R. M., Saiz, E., Tomsia, A. P. and Carter, W. C. in Materials Research Symposium Proceedings, Vol 357: Structure and Properties of Interfaces in Ceramics, eds. D.A. Bonnell, M. Rühle, and U. Chowdhry, MRS. Pittsburgh, 1995, 279.

44. Bikerman, J. J., Physical Surfaces. 1970, New York: Academic Press. 97-110, 128-31.

45. Harkins, W. D., The Physical Chemistry of Surface Films. 1952, New York: Reinhold Publishing Corp. pf. 175.

46. Sharps, P. R., Tomsia, A. P., and Pask, J. A., Acta Met., 1981, 29, 855.

47. Ghetta, V., Fouletier, J. and Chatain, D., Acta. Mater., 1996, 44, 1927.

48. Merlin, V. and Eustathopoulos, N., J. Mater. Sci., 1995, 30, 3619.

49. Wang, D.-J. and Wu, S.-T., Acta Metall. Mater., 1995, 43, 2917.

50. Köstlmeier, S. and Elsässer, C., Density Functional Study of the "Titanium Effect" at Metal Ceramic Interface, to be published.

51. Bikerman, J. J., J. Phys. Chem., 1959, 63, 1658.

52. Saiz, E., Tomsia, A. P. and Cannon, R. M., Acta Mater., 1998, 46, 2349.

53. Shanahan, M. E. R. and Carre A., Langmuir, 1995, 11, 1396.

54. Saiz, E., Cannon R. M., and Tomsia, A. P., submitted to Scripta Mater.

55. Mullins, W. W., J. Appl. Phys., 1957, 28, p. 333.

56. Mullins, W. W., Acta Met., 1958, 6, 414.

57. Saiz, E., Cannon, R. M. and Tomsia, A. P., Acta Mater., 1999, 47, 4209.

58. Saiz, E., and Tomsia, A. P., J. Amer. Ceram. Soc., 1998, 81, 2381.

59. Turnbull, D., Solid State Physics. 1956, New York, Academic Press.

60. Christian, J. W., Ch 10, in Theory of Transformations in Metals and Alloys. 1975, Pergamon Press, New York. p. 418.

61. Pandit, R., Schick, M., and Wortis, M., Phys. Rev. B, 1982, 26, 5112.

62. Schick, M., in Liquids at Interfaces, Les Houches Summer School Lectures, Session XL VIII, eds. J. Charvolin, J. F. Joanny, and J. Zinn-Justin, Elsevier, Amsterdam, 1989.

63. Dietrich, S., in Phase Transitions in Surface Films, ed. H. E. A. Taub,, Plenum Press: New York., 1991, p. 391.

64. Kim, W. T. and Cantor, B., Acta Met. Mat., 1994, 42, 3115.

65. Laurent, V., Chatain, D. and Eustathopoulos, N., Mater. Sci. Eng., 1991, A135, 89.

66. Meier, A., Chidambaram, P. R. and Edwards, G. R., Acta Mat., 1998, 46, 4453.

67. Boettinger, W. J., Handwerker, C. A.. and Kattner, U. R., in The Mechanics of Solder Alloy Wetting and Spreading, eds. F. G. Yost, F. M. Hosking, and D. R. Frear, Van Nostrand Reinhold, New York., 1993, 103.

68. Pesach, D. and Marmur, A., Langmuir, 1987, 3, 519.

69. DeVincent, S. M.. and. Michal, G. M., Met. Trans. A, 1993, 24, 53.

70. Nogi, K., Osugi, Y., and Ogino, K., Iron Steel Institute of Japan, 1990, 30, 64.

71. Diemer, M., Neubrand, A., Trumble, K. P., and Rödel, J., J. Am. Ceram. Soc., 1999, 82, 2825. 


\section{FIGURE CAPTIONS}

Figure 1. Effects of Ti additions on wetting angles on $\mathrm{Al}_{2} \mathrm{O}_{3}$ for several metallic alloys. Data for $\mathrm{Cu}-\mathrm{Ti}$ are from Refs. [7, 14-18]; for eutectic $\mathrm{Ag}-\mathrm{Cu}$ from [11, 19]; and for Sn, from [16, 20].

Figure 2. Spreading kinetics of different high-temperature metallic liquids, typically between those shown for $\mathrm{Ag}-\mathrm{Cu}-\mathrm{Ti}$ and $\mathrm{Cu}-\mathrm{Nb}$ on $\mathrm{Al}_{2} \mathrm{O}_{3}$, are far slower than for low viscosity organic or aqueous liquids, which exhibit rates similar to or faster than those shown for glycerol-water [25]. However, recently reported results from drop transfer experiments $[23,24]$ yield much higher speeds. All data are for advancing fronts.

Figure 3. Reactive wetting requires several steps - from fluid flow, adsorption and triple line ridging, to formation of a phase that may finally cover the solid-liquid interface. With a large nucleation barrier, a time regime exists in which reaction lags behind the front; contact angles are then dictated by adsorption at the metastable interfaces, and spreading rates can be limited by ridge drag. Occasionally, the front can be dislodged from the reaction product and the sequence repeats.

Figure 4. Continuum (a) versus atomic (b) approaches for the analysis of spreading (after Refs. [34] and [32], respectively). Hydrodynamic analyses have recognized that the classical no-slip condition at the wall boundary causes an unbounded free singularity at the triple line (the force required to advance the liquid becomes logarithmically infinite) and should be relaxed.

Figure 5. Expected relationship between contact angle and spreading velocity for liquids spreading under regime I. A unique dynamic contact angle associated with each spreading velocity may be expected for smooth, homogeneous surfaces, although different stationary angles for advancing and receding are often indicated experimentally.

Figure 6. Hypothetical variation of the interface energies with the activity of a reactive element $\left(a_{R}\right)$ for a system in which that element adsorbs on all three interfaces involved. The corresponding variation of contact angle is also depicted, with open symbols corresponding to the dry spreading limit, based on a nonequilibrium situation in which (for dynamic reasons) no prior adsorption has occurred at the solid surface and the value for a clean surface $\left(\gamma_{s v}^{o}\right)$ pertains for all activities of the reactive element.

Figure 7. Evolution of contact angles for initially advancing and receding fronts for $\mathrm{Cu}-1.4$ at $\%$ $\mathrm{Nb}$ on $\mathrm{Al}_{2} \mathrm{O}_{3}$. Angles for the two types of fronts diverge. Although not shown for the data plotted, the values of $\theta$ actually crossed for the two types of $\mathrm{Cu}-\mathrm{Nb}$ experiments at very short times; that is, the initial values of $\theta$ were smaller for the receding drops for which the angles eventually became larger.

Figure 8. Expected relationships between contact angle and spreading velocity for liquids spreading under regime II. The relation is not unique; there is a $\theta$ interval for which a broad range of possible spreading velocities exist which are inversely proportional to the ridge height.

Figure 9. AFM image of ridge that developed at the triple junction of a receding $\mathrm{Cu}$ drop on sapphire after $150 \mathrm{~min}$ at $1150^{\circ} \mathrm{C}$ in gettered Ar.

Figure 10. Possible triple line configurations, with their corresponding wetting driving forces and spreading kinetics. 
Figure 11. Sketch showing how the interfacial energy varies for three different forms of interfacial adsorption. The three situations will exhibit markedly different barriers for heterogeneous nucleation of a new phase at the interface.

Figure 12. SEM images of cross sections of two wetting specimens of eutectic $\mathrm{Ag}-\mathrm{Cu}+1.9 \%$ $\mathrm{Ti} / \mathrm{Al}_{2} \mathrm{O}_{3}$ after annealing at $1000^{\circ} \mathrm{C}$ for 1 hour in $\mathrm{Ar}$. In (a), the drop on sapphire spread to $\theta \sim 20^{\circ}$, and a uniform layer of $\mathrm{TiO}$-rich reaction product formed at the interface. The other sample (b), made with $96 \% \mathrm{Al}_{2} \mathrm{O}_{3}$, is revealing; for this, the liquid was arrested at a higher angle. A uniform layer of TiO formed, but then the front dislodged and advanced further, and again subsequently formed thin layers of reaction product at the interface, as seen in the higher magnification images below.

Figure 13. Evolution of contact angles showing the different behavior of initially advancing and receding fronts for Sn-Ti on sapphire. The angles for receding and advancing drops exhibit some persistent hysteresis for two different types of samples. 